\title{
Determination of Ghrelin’s Role in the Pathogenesis of Pregnancy Induced Hypertension
}

Izabela Ulman-Włodarz'1,3, Tomasz Irzyniec ${ }^{2,4 *}$, Ewa Galbierz- Kwiatkowska ${ }^{2}$ and Izabela Maciejewska-Paszek ${ }^{2}$

${ }^{1}$ Faculty of Health Sciences University of Bielsko Biała, Poland

${ }^{2}$ Department of Health Promotion and Community Nursing, Medical University of Silesia, Katowice, Poland

${ }^{3}$ Department of Obstetrics and Gynecology, MEGRES Hospital, Tychy, Poland

${ }^{4}$ Department of Nephrology/ENDO MSW Hospital, Katowice, Poland

\begin{abstract}
Background: Ghrelin is a stomach-produced polypeptide hormone that stimulates hunger and secretion of growth hormone. Abnormalities in ghrelin secretion are well documented in obese patients, but explored little in patients with various forms of hypertension. Notably, ghrelin is indirectly involved in blood pressure regulation in pregnancy induced hypertension.

Material and methods: The present study aimed to assess ghrelin concentration in venous blood of 19 nonpregnant (NP), 20 healthy pregnant (HP) and 20 hypertensive pregnant (PIH). It also included assessing blood ghrelin concentration of 21 obese hypertensive pregnant (OPIH) women before and one hour after eating. Ghrelin secretion was estimated 1-3 days before delivery.

Results: All experimental groups of pregnant women (HP, PIH, and OPIH) showed significantly reduced ghrelin plasma level as compared with NP $(812 \pm 83,716 \pm 66$, and $717 \pm 52$ vs $1057 \pm 74$, respectively). We found no significant ghrelin reduction after eating in obese hypertensive pregnant women. The blood ghrelin concentration in pregnant women negatively correlated with mean arterial pressure. In spite of the differences in ghrelin concentration in women, whom a decrease in ghrelin concentration after a standard meal was observed compared to women, who didn't reduce of ghrelin secretion no differences were found in mean arterial pressure.
\end{abstract}

Conclusion: The relationship between arterial hypertension and ghrelin secretion disorders during hypertensive pregnancy is uncertain and requires further examination.

Keywords: Ghrelin; Pregnancy; Hypertension; Obesity

\section{Introduction}

Pregnancy induced hypertension (PIH) is a disease of an unclear etiology that complicates $2-3 \%$ of pregnancies. PIH is a critical cause of perinatal mortality of pregnant women and neonates and a major cause of intrauterine growth retardation and of iatrogenic prematurity [1]. PIH is a multiorgan complication; its main causes result from the interaction of the mother's immunological system with trophoblast antigens. The ethiopathogenesis of pregnancy induced hypertension remains to be elucidated.

A crucial risk factor of pregnancy induced hypertension is obesity. Relationships between hypertension and obesity are well documented [2], ultimately involving excessive retention of sodium by the kidneys, insulin resistance, and chronic stimulation of the sympathetic system, leading to vessel remodelling. Leptin levels are increased in obese patients, stimulating the proliferation of smooth muscles cells of arterial vessels and influencing Renin-Angiotensin-Aldosterone System activity. Pregnant women experience excessive appetite, which leads to overall body mass increase [3].

Ghrelin, a hormonemainly produced in the digestive tract, isinvolved in releasing growth hormone, increasing appetite, and inducing obesity. Ghrelin stimulates the secretion of prolactin and adrenocorticotrophic hormone and has a crucial impact on steroidogenesis and carbohydrate metabolism [4]. Disorders surrounding adrenocorticotrophic hormone secretion are observed in obese individuals. Results regarding changes in ghrelin secretion in various types of hypertension, however, are equivocal. Ghrelin is known to play a crucial role in the intrauterine growth of the fetus; blood ghrelin concentration in intrauterine growth retardation fetuses has been reported to be higher than in healthy ones [5].
Based on the possible relationship between hypertension and obesity in pregnant women together with the change in ghrelin secretion in obese individuals, we sought to discern whether ghrelin governs related secretion disorders and plays a role in the ethiopathogenesis of pregnancy induced hypertension.

We sought to answer the following questions:

TIt is evident that our research has both scientific and practical aspects. Showing a relationship between ghrelin secretion and hypertension and/or obesity would prove that ghrelin analysis is a useful tool in evaluating the fetal condition and/or pregnancy prognosis.

\section{Materials and Methods}

Our study included 61 pregnant women. The first group consisted of 20 healthy pregnant (HP), the second of 20 hypertensive pregnant women (PIH), and the third of 21 pregnant women with hypertension and obesity (OPIH). The mean ages of the groups were: $28.1 \pm 1.1,25.7$ \pm 1.2 and $27.5 \pm 1.1$ years respectively. Differences between the mean

*Corresponding author: Tomasz Irzyniec, Medical University of Silesia Department of Health Promotion and Community Nursing ul. Głowackiego 1040 - 052 Katowice, Poland, Tel: +48 32 782-72-21; Fax: +48 32 782-73-00; E-mail: tirzyniec@sum.edu.pl

Received April 25, 2015; Accepted May 30, 2015; Published June 06, 2015

Citation: Ulman-Włodarz I, Irzyniec T, Galbierz- Kwiatkowska E, MaciejewskaPaszek I (2015) Determination of Ghrelin's Role in the Pathogenesis of Pregnancy Induced Hypertension. J Hypertens 4: 201. doi:10.4172/2167-1095.1000201

Copyright: (c) 2015 Ulman-Włodarz I, et al. This is an open-access article distributed under the terms of the Creative Commons Attribution License, which permits unrestricted use, distribution, and reproduction in any medium, provided the original author and source are credited. 
ages were not significant. All women gave birth to healthy children with Apgar scores between 7 and 10 points. The control group, consisting of 19 healthy non-pregnant women at the age of $20.2 \pm 0.1$ (NP), was also examined. We received a statement on informed consent from the study participants. The study protocol was approved by the Bioethical Commission of the Medical University of Silesia. Pregnant women (unifetal pregnancies) with both hypertension and other systemic diseases (including diabetes) were not included to the study. Blood from each woman was drawn from the basilic vein after a fasting period 2-3 days before delivery. Haemoglobin, glucose, protein, creatinine, and uric acid concentrations were determined. Plasma ghrelin concentration was assessed before and one hour after the intake of a standard breakfast. All patients had had their arterial blood pressure measured before blood was drawn.

The mean arterial blood pressure (MAP) was determined according to the formula:

$\mathrm{MAP}=$ diastolic pressure $+($ systolic pressure-diastolic pressure $) / 3$.

Body Mass Index (BMI) was calculated according to the formula:

BMI $=$ body mass $/$ height $^{2}\left(\mathrm{~kg} / \mathrm{m}^{2}\right)$

A radio immunological method was used to determine ghrelin concentration using the Total Ghrelin Ria Kit. The standard breakfast included: $40 \mathrm{~g}$ of cornflakes, $250 \mathrm{ml}$ of milk, $100 \mathrm{ml}$ of grapefruit juice, and $5 \mathrm{~g}$ of sugar, totaling an energetic value of $369.6 \mathrm{kcal}(1545 \mathrm{~kJ})$.

Results are presented as mean \pm SEM. The distribution normality was assessed using the Kołmogorow-Smirnow test. A student's t-test was employed to determine the mean for dependent and independent variables. The U-Mann Whitney test was used to analyze ghrelin concentration data. Occurrence correlations were evaluated using aPearson's linear correlation and $\tau$ Kendal test (for Ghrelin). Differences were considered statistically significant at $\mathrm{p}<0.05$.

\section{Results}

Hypertensive groups of pregnant women expressed significantly increase MAP compared both, to values at the beginning of pregnancy -PIH: $114 \pm 2$ vs. $96 \pm 1 \mathrm{mmHg}$ and OPIH: $119 \pm 3$ vs. $99.8 \pm 1$ $\mathrm{mm} \mathrm{Hg}-\mathrm{p}<0,01$ and to healthy pregnant ones HP: $92 \pm 1$ vs. $90 \pm$ $1 \mathrm{mmHg}-\mathrm{p}<0,01$, respectively. Clinical and laboratory characteristics of studied populations are presented in Table 1. Our results revealed significantly lower plasma ghrelin concentrations in all pregnant women in comparison with non pregnant ones. Ghrelin concentration significantly decreased after a standard meal in normal pregnant women; however, this trend did not occur in OPIH group members (Table 2).

Based on the $\tau$ Kendall correlation coefficient, a negative correlation was found between the random variables BMI and ghrelin in the population of healthy pregnant women (HP) for the assumed level of significance $\alpha, \mathrm{p}<0.05$.

As a result of the statistical analysis for the Pearson's linear correlation testa positive correlations between BMI and MAP and BMI and glucose were found in hypertensive pregnant women $(\mathrm{PIH}+\mathrm{OPIH})$ $\mathrm{p}=0.036$ and $\mathrm{p}<0.001$ respectively.

Based on Pearson's linear correlation test, a positive correlations were found between both the random variables BMI and MAP as well as BMI and glucose in the whole population of pregnant women $(\mathrm{HP}+\mathrm{PIH}+\mathrm{OPIH})$ for assumed levels of significance $\alpha, \mathrm{p}<0.001$. Additionally, as a result of the statistical analysis for the $\tau$ Kendall correlation coefficient, a negative correlation between the random variables MAP and ghrelin was found in this population for the assumed relevance level $\alpha, p=0.043$. The calculated correlation coefficient $\tau_{\mathrm{K}}$ is -0.26 , which means that the dependence level between the analyzed statistical characteristics in the probe taken from the subject general population was low.

In pregnant women, whom a decrease in ghrelin concentration after a standard meal was observed $(\mathrm{n}=43)-846.4 \pm 44.8 \mathrm{ng} / \mathrm{ml}$ compared to pregnant women, who didn't reduce of ghrelin secretion after standard breakfast $(\mathrm{n}=18)-513.5 \pm 40.5 \mathrm{ng} / \mathrm{ml}$, no differences were found in mean arterial pressure $109 \pm 2$ vs $109 \pm 1 \mathrm{mmHg}$.

\section{Results Summary}

Our results shows a standard clinical and biochemical picture of pregnancy characterized by an increase in body mass and BMI, a decrease in hemoglobin level, and a decrease in creatinine and protein concentrations in all pregnant subjects as compared to non pregnant ones. Hypertensive pregnant women (PIH and OPIH) exhibited higher blood pressure during pregnancy. Our results revealed significantly lower plasma ghrelin concentrations in all pregnant women in comparison with non pregnant ones.

$\mathrm{PIH}$ differed in women with uncomplicated pregnancies with no negative correlation between ghrelin concentration and BMI. Additionally, no difference in glucose, creatinine, and protein concentration was noted. OPIH differed in women with uncomplicated pregnancies by the significance in glycaemia and uricaemia. No significant ghrelin decrease after a standard meal was observed. The $\mathrm{OPIH}$ women differed from the PIH women by higher BMI and glycaemia, but not by blood pressure.

A negative correlation between mean arterial pressure and ghrelin concentration was reported in all pregnant women together. In spite of the aforementioned differences in ghrelin concentration in women, whom a decrease in ghrelin concentration after a standard meal was observed compared to women, who didn't reduce of ghrelin secretion no differences were found in mean arterial pressure.

\section{Discussion}

Hypertension is a frequent complication of pregnancy, and the ethiopathogenesis is multifactorial. Generalized blood vessel contraction, which induces vascular resistance, is the basic pathophysiological phenomenon. These contractions can have destructive impact on vessels. Hypertension in pregnancy is associated with blood vessel sensitivity increases to substances of pressure activity [3].

Many pathogenetic foci are listed, including genetically determined parameters that also may contribute to blood pressure increases during pregnancy. Important contributors to developing arterial hypertension during pregnancy are environmental factors [6]. The impacts of body mass increases during pregnancy and insufficient nutrients for the mother and fetus, including arterial hypertension, contribute to etiopathogenesis. It is important than women control body mass gain because an excess can result in hypertension [7]. The impact of obesity in the etiopathogenesis of arterial hypertension is undeniable [8]. Both obesity and arterial hypertension in pregnant women are known to lead to uteroplacental blood flow impairment [9], resulting in changes in placenta morphology, ultimately decreasing the active surface of the organ.

Ghrelin influences the energy regulation of the body. Ghrelin is 
Citation: Ulman-Włodarz I, Irzyniec T, Galbierz- Kwiatkowska E, Maciejewska-Paszek I (2015) Determination of Ghrelin’s Role in the Pathogenesis of Pregnancy Induced Hypertension. J Hypertens 4: 201. doi:10.4172/2167-1095.1000201

Page 3 of 5

\begin{tabular}{|c|c|c|c|c|}
\hline & NP $n=19$ & $\mathrm{HP} n=20$ & PIH n=20 & OPIH $n=21$ \\
\hline Body mass [kg] & $58,9 \pm 0,9(55-70)$ & $75,6 \pm 2,2 \#(62-95)$ & $75,3 \pm 1,6 \#(62-90)$ & $104,3 \pm 4,2 \# !(79-156)$ \\
\hline $\mathrm{BMI}\left[\mathrm{kg} / \mathrm{m}^{2}\right]$ & $20,7 \pm 0,2(19,6-23,7)$ & $27,1 \pm 0,4 \#(23-31,5)$ & $28,0 \pm 0,6 \#(23,5-31,1)$ & $37,8 \pm 1,2 \# !(32,3-52,7)$ \\
\hline Mean arterial pressure $[\mathrm{mmHg}]$ & $90,4 \pm 1,4(83-102)$ & $91,9 \pm 1,2(83-103)$ & $114,3 \pm 1,9 \#(100-133)$ & $119,4 \pm 2,6 \#(97-150)$ \\
\hline Hemoglobin [g/dl] & $13,7 \pm 0,2(11,8-15,4)$ & $12,1 \pm 0,3 \#(9-13,3)$ & $12,6 \pm 0,3 \#(10,1-15,9)$ & $11,9 \pm 0,3 \#(9,4-14,1)$ \\
\hline Glucose $[\mathrm{mmol} / \mathrm{l}]$ & $4,3 \pm 0,1(3,8-4,7)$ & $4,0 \pm 0,1^{*}(2,4-5)$ & $3,9 \pm 0,1^{*}(2,8-5)$ & $4,6 \pm 0,2(3,9-6,5)$ \\
\hline Creatinine $[\mu \mathrm{mol} / \mathrm{l}]$ & $72 \pm 2(59-85)$ & $52 \pm 1,9 \#(35-65)$ & $55, \pm 2,7 \#(41-81)$ & $53,4 \pm 2,1 \#(41-76)$ \\
\hline Protein $[\mathrm{g} / \mathrm{l}]$ & $81,1 \pm 1,4(75-94)$ & $65,5 \pm 1,4 \#(55-75,7)$ & $63,2 \pm 1,3 \#(52-76)$ & $63,8 \pm 1,1 \#(55,3-71,5)$ \\
\hline Uric acid $[\mu \mathrm{mol} / \mathrm{l}]$ & $273 \pm 11(160-357)$ & $290 \pm 16(60-353)$ & $336 \pm 16 \#^{\wedge}(229-527)$ & $335 \pm 16 \#^{\wedge}(171-465)$ \\
\hline
\end{tabular}

Table 1: Clinical and laboratory characteristics of the studied populations of non-pregnant (NP), healthy pregnant (HP) as well as hypertensive (PIH) and obese, hypertensive (OPIH) pregnant women. mean \pm SEM (range).

\begin{tabular}{|c|c|c|c|}
\hline & $\begin{array}{c}\text { Ghrelin (before) } \\
{[\mathrm{ng} / \mathrm{ml}]}\end{array}$ & $\begin{array}{c}\text { Ghrelin (after) } \\
\text { [ng/ml] }\end{array}$ & before vs after \\
\hline NP & $\begin{array}{c}1056,6 \pm 74,1 \\
(663-1808)\end{array}$ & $\begin{array}{c}910,9 \pm 51,7 \\
(530-1370)\end{array}$ & $p<0,001$ \\
\hline $\begin{array}{c}\text { HP } \\
\text { a.c. NP. }\end{array}$ & $\begin{array}{c}811,8 \pm 83,0 \\
(417-1988) \\
p=0,0347\end{array}$ & $\begin{array}{c}680,6 \pm 49,4 \\
(311-1107) \\
p=0,0026\end{array}$ & $p<0,001$ \\
\hline $\begin{array}{l}\text { PIH } \\
\text { a.c. NP } \\
\text { a.c. HP }\end{array}$ & $\begin{array}{c}716,4 \pm 65,7 \\
(302-1520) \\
p=0,0014 \\
\text { NS }\end{array}$ & $\begin{array}{c}\mathbf{6 3 4 , 8} \pm \mathbf{5 5 , 3} \\
\mathbf{( 3 3 3 - 1 4 6 0 )} \\
p=0,0008 \\
\text { NS }\end{array}$ & $p=0,0472$ \\
\hline $\begin{array}{l}\text { OPIH } \\
\text { a.c. NP } \\
\text { a.c. HP } \\
\text { a.c. PIH }\end{array}$ & $\begin{array}{c}\mathbf{7 1 7 , 8} \pm \mathbf{5 2 , 3} \\
(\mathbf{3 8 0}-1234) \\
p=0,0005 \\
\text { NS } \\
\text { NS }\end{array}$ & $\begin{array}{c}645,1 \pm 44,3 \\
(354-1207) \\
p=0,0004 \\
\text { NS } \\
\text { NS }\end{array}$ & NS \\
\hline
\end{tabular}

Table 2: Plasma ghrelin concentration before and after standard breakfast in studied populations of women. mean \pm SEM (range)

a.c. - statisticaly significant as compared to group

NS - not statisticalysiginficant

strictly associated with metabolic conversions, showing multidirectional activity by acting both endocrinally and paracrinally [10]. Changes in ghrelin concentrations in patients with both arterial hypertension and obesity are currently the subject of intensive studies [11,12], but little examinations regarding ghrelin and hypertensive or obese pregnant women exists.

Pregnancy regularities in all the groups were confirmed by a lack of differences in Apgar score mean values of newborns. Pregnancy induced hypertension patients exhibited normal arterial pressure at the beginning of pregnancy. Obesity may have onset before pregnancy because the weight increases during pregnancy were comparable among all groups. Ghrelin concentrations caused by a standard meal under both basic conditions and secretion were monitored.

Both clinical and biochemical parameters affirmed that all women underwent typical pregnancies characterized by weight and BMI increases, a decrease in hemoglobin content, and decreases in creatinine and blood serum protein concentrations. Differences between healthy pregnant women and pregnant women with hypertension, regarding weight, body mass index, glycaemia, and uricaemia in the OPIH group, seem to be the consequence of pre-pregnancy obesity. We found lower ghrelin concentrations in all groups of pregnant women compared to women who were not pregnant. Similar results were also reported by Riedl [13]. Low plasma ghrelin is associated with insulin resistance, hypertension, and the prevalence of type 2 diabetes was observed by Pöykkö [11].
The reason for these lower ghrelin levels remains to be determined.

The lack of significant differences in ghrelin concentrations in hypertensive groups does not exclude it as influencing the etiopathogenesis of hypertension. The central and peripheral activities of ghrelin include stimulating both hunger feelings and motoric activity of the alimentary tract, and initiating eating. An increase in blood ghrelin concentration precedes a meal. Limiting food intake and following a low protein diet leads to greater ghrelin secretion. Conversely, a high-fat and, carbohydrate-rich diet hampers its secretion. Kawamura et al. [14] showed that ghrelin levels significantly rise during starvation. Additionally, ghrelin levels in slim individuals increases progressively before each meal and decreases just one hour after it [15]. Firczykcharacterized hemodialysed patients by higher ghrelin concentrations than the control group [16]. We report a significant decrease in ghrelin concentration after a standard meal in pregnant women. This normal trend, however, fails to appear in pregnant women with arterial hypertension and obesity.

During pregnancy, a negative correlation between BMI and ghrelin concentration has been observed. Again, we failed to find this correlation in pregnant women with hypertension, both in the PIH and OPIH groups. We conclude that arterial hypertension in pregnant women complicates these relationships.

In patients with polycystic ovary syndrome, the ghrelin levels correlate negatively with BMI, a trend opposing that in healthy women $[17,18]$. 
Jołda-Mysłowska [19] determined ghrelin concentrations in patients with idiopathic arterial hypertension, finding that concentrations in blood serum in these patients were lower than control. Makino et al. [5] obtained different results, reporting that patients with pregnancy induced hypertension exhibited higher ghrelin levels. Additionally, they noted a positive correlation between ghrelin concentration and arterial pressure in these patients, and a positiverelationship between ghrelin and blood pressure in healthy pregnant women, which was not observed in non pregnant ones.

The relationship between ghrelin, insulinemia, and insulin resistance in obese hypertension patients was examined by Dytfeld et al. [20]. Decreased concentrations of ghrelin in blood serum were observed in obese individuals with hypertension. Body mass reduction was associated with an increase in ghrelin concentration [17].

Prerequisites exist regarding the extent of ghrelin secretion disorders in the etiopathogenesis of arterial hypertension. Although $\mathrm{BMI}$ and MAP as well as BMI and glucose concentrations were correlated, ghrelin concentration was only found to correlate with mean arterial pressure. Thus, it cannot be unequivocally presumed whether ghrelin deficiency is responsible for arterial hypertension or vice versa (that the occurrence of hypertension modifies ghrelin secretion). Our reported negative relationship between mean arterial pressure and ghrelin concentration in all pregnant subjects suggests an etiopathogenetic link between pregnancy induced hypertension and ghrelin secretion.

Makino et al. [5] claimed that ghrelin levels are lower in obese patients, and a negative correlation between ghrelin concentration and BMI exists. A ghrelin level decline in the third trimester of pregnancy was described by Fuglsang et al. [21], which supported Makino's result, showing that women reach their greatest body mass at the end of the pregnancy period.

Considering the impact of ghrelin on glucose metabolism, its influence on insulin resistance stimulation, obesity, and GH secretion, which by its anti-insulin activity increases glucose concentration in blood serum, should be highlighted. This peripheral activity of ghrelin in glucose metabolism has been confirmed in the literature [22].

Standard meal consumption allowed for monitoring hormonal mechanisms, including ghrelin secretion disorders, which can potentially influence arterial pressure. During a standard pregnancy, a standard meal causes ghrelin concentration to significantly decrease; the lack of such a decrease in hypertensive pregnant women with obesity can foster etiopathogenetic prerequisites for hormone secretion. Whether this hormone configuration is typical for the obese in the hypertensive group of pregnant women, requires further investigation. Notably, the lack of the aforementioned correlation in the hypertensive groups (PIH, and OPIH), stands against a connection between arterial hypertension and ghrelin secretion disorders.

No mean arterial pressure difference between groups exhibiting decreased ghrelin concentration was seen after a standard meal. This data also argue against a close dependence of ghrelin secretion changes and arterial hypertension.

It can thus be stated that the relationship between arterial pressure values and ghrelin secretion disorders in hypertensive pregnant women is uncertain and requires further investigation.

Hypertension during pregnancy remains problematic. Due to complications of hypertension, relevant investigations revolve around various scientific centers. Suggested procedure algorithms prove that a current, constant search for optimal diagnostics and therapy are underway. The discovery of novel hormone activity will enable recognition of a pregnancy-induced hypertension mechanism.

\section{Conclusions}

1. Ghrelin concentration in the blood serum of pregnant women is lower than in non-pregnant women.

2. Women with pregnancy induced hypertension and obesity differ from healthy pregnant women in food intake-induced change of ghrelin secretion.

3. The relationship between arterial hypertension and ghrelin secretion disorders during hypertensive pregnancy is uncertain and requires further examination.

\section{Disclosure Statement}

The authors have nothing do disclose.

\section{References}

1. Magee LA, Helewa M, Moutquin JM, von Dadelszen P; Hypertension Guideline Committee; Strategic Training Initiative in Research in the Reproductive Health Sciences (STIRRHS) Scholars (2008) Diagnosis, evaluation, and management of the hypertensive disorders of pregnancy. J Obstet Gynaecol Can 30: S1-48.

2. Kang YS (2013) Obesity associated hypertension: new insights into mechanism Electrolyte Blood Press 11: 46-52.

3. Mugo M, Govindarajan G, Kurukulasuriya LR, Sowers JR, McFarlane SI (2005) Hypertension in pregnancy. Curr Hypertens Rep 7: 348-354.

4. Klok MD, Jakobsdottir S, Drent ML (2007) The role of leptin and ghrelin in the regulation of food intake and body weight in humans: a review. Obes Rev 8 : 21-34.

5. Makino Y, Hosoda H, Shibata K, Makino I, Kojima M, et al. (2002) Alteration of plasma ghrelin levels associated with the blood pressure in pregnancy. Hypertension 39: 781-784.

6. Khalil A, Jauniaux E, Harrington K (2009) Antihypertensive therapy and centra hemodynamics in women with hypertensive disorders in pregnancy. Obstet Gynecol 113: 646-654

7. Wolfe H (1998) High prepregnancy body-mass index--a maternal-fetal risk factor. N Engl J Med 338: 191-192.

8. Kshatriya S, Liu K, Salah A, Szombathy T, Freeman RH, et al. (2011) Obesity hypertension: the regulatory role of leptin. Int J Hypertens 2011: 270624.

9. Stothard KJ, Tennant PW, Bell R, Rankin J (2009) Maternal overweight and obesity and the risk of congenital anomalies: a systematic review and metaanalysis. JAMA 301: 636-650.

10. Kojima M, Hosoda H, Date Y, Nakazato M, Matsuo H, et al. (1999) Ghrelin is a growth-hormone-releasing acylated peptide from stomach. Nature 402 : 656-660.

11. Pöykkö SM, Kellokoski E, Hörkkö S, Kauma H, Kesäniemi YA, et al. (2003) Low plasma ghrelin is associated with insulin resistance, hypertension, and the prevalence of type 2 diabetes. Diabetes 52: 2546-2553.

12. Rodríguez A, Gómez-Ambrosi J, Catalán V, Becerril S, Sáinz N, et al. (2010) Association of plasma acylated ghrelin with blood pressure and left ventricular mass in patients with metabolic syndrome. J Hypertens 28: 560-567.

13. Riedl M, Maier C, Handisurya A, Luger A, Kautzky-Willer A (2007) Insulin resistance has no impact on ghrelin suppression in pregnancy. J Intern Med 262: 458-465.

14. Kawamura K, Sato N, Fukuda J, Kodama H, Kumagai J, et al. (2003) Ghrelin inhibits the development of mouse preimplantation embryos in vitro. Endocrinology 144: 2623-2633.

15. Otto Buczkowska $E$ (2005) [The role of ghrelin in the regulation of energy homeostasis]. Endokrynol Diabetol Chor Przemiany Materii Wieku Rozw 11: 39-42.

16. Firczyk P, Ignacy W, Adamczak M, Chudek J, Wiecek A (2009) Postprandial 
Citation: Ulman-Włodarz I, Irzyniec T, Galbierz- Kwiatkowska E, Maciejewska-Paszek I (2015) Determination of Ghrelin’s Role in the Pathogenesis of Pregnancy Induced Hypertension. J Hypertens 4: 201. doi:10.4172/2167-1095.1000201

Page 5 of 5

secretion of ghrelin in hemodialysis patients with and without diabetic nephropathy. Pol Arch Med Wewn 119: 447-452.

17. Orio F Jr, Lucidi P, Palomba S, Tauchmanova L, Cascella T, et al. (2003) Circulating ghrelin concentrations in the polycystic ovary syndrome. J Clin Endocrinol Metab 88: 942-945.

18. Schöfl C, Horn R, Schill T, Schlösser HW, Müller MJ, et al. (2002) Circulating ghrelin levels in patients with polycystic ovary syndrome. J Clin Endocrinol Metab 87: 4607-4610.

19. Jolda-Mydlowska B, Kobusiak-Prokopowicz M, Przewlocka-Kosmala M Witkowska M Urotensin II and ghrelin and target organ damage in primary hypertension. (2006) ArterialHypertension 10: 128-135.

20. Dytfeld J, Pupek-MusialikD (2007) Ghrelin and its relationship with insulin and insulin resistance in obese hypertensive patients. PrzegKardiodiabetol 2: 27 34.

21. Fuglsang J, Skjaerbaek C, Espelund U, Frystyk J, Fisker S, et al. (2005) Ghrelin and its relationship to growth hormones during normal pregnancy. Clin Endocrinol (Oxf) 62: 554-559.

22. Suzuki K, Jayasena CN, Bloom SR (2012) Obesity and appetite control. Exp Diabetes Res 2012: 824305. 